\title{
Unconventional Low-Cost Fabrication and Patterning Techniques for Point of Care Diagnostics
}

\author{
Himanshu Sharma, ${ }^{1}$ Diep Nguyen, ${ }^{2}$ Aaron Chen, ${ }^{1}$ Valerie Lew, ${ }^{2}$ and Michelle Khine ${ }^{1,2}$ \\ ${ }^{1}$ Department of Chemical Engineering \& Materials Science, University of California, Irvine, CA, USA; and ${ }^{2}$ Department \\ of Biomedical Engineering, University of California, Irvine, CA, USA
}

(Received 16 September 2010; accepted 17 November 2010; published online 9 December 2010)

Associate Editor Daniel Takashi Kamei oversaw the review of this article.

\begin{abstract}
The potential of rapid, quantitative, and sensitive diagnosis has led to many innovative 'lab on chip' technologies for point of care diagnostic applications. Because these chips must be designed within strict cost constraints to be widely deployable, recent research in this area has produced extremely novel non-conventional micro- and nano-fabrication innovations. These advances can be leveraged for other biological assays as well, including for custom assay development and academic prototyping. The technologies reviewed here leverage extremely low-cost substrates and easily adoptable ways to pattern both structural and biological materials at high resolution in unprecedented ways. These new approaches offer the promise of more rapid prototyping with less investment in capital equipment as well as greater flexibility in design. Though still in their infancy, these technologies hold potential to improve upon the resolution, sensitivity, flexibility, and cost-savings over more traditional approaches.
\end{abstract}

Keywords-Point of care, Lab on a chip, Immunoassay, Microfabrication, Surface plasmon resonance, Nanofabrication.

\section{INTRODUCTION}

At the interface between fundamental academic research and the global demand for low-cost, translational biomedical technology lays an exciting bridge that has led to many recent significant advances in point of care (POC) diagnostics. ${ }^{46,93}$ Since the 1970s, the development of POC technologies can be seen in such devices as glucose and urine sensors which have been miniaturized and adopted for household use. ${ }^{28}$ In short, these tests can be administered at a patient's locale and even by the patient himself, offering not only convenience but significantly more rapid diagnosis than conventional

Address correspondence to Michelle Khine, Department of Chemical Engineering \& Materials Science, University of California, Irvine, CA, USA. Electronic mail: mkhine@uci.edu lab-based testing. Reducing the time to diagnosis from days to minutes enables better patient management decisions that may lead to improved patient compliance, prognosis and reduced overall cost of care.

Within this past decade, lab on chip (LOC) growth has been driven by its numerous advantages over macroscale devices including faster reaction time, less reagent and sample consumption, portability, and lower capital equipment costs. ${ }^{4,17,67,73,88}$ Advantages gained from microfabrication and nanofabrication approaches have since been leveraged for chemical, biological, and physical processes from the molecular to the cellular scales. Applications of such microfluidic-based LOC devices include fractionation, mixing, purification, reactions, separations, and detections. ${ }^{9,23}$ These technologies have the potential to simplify and improve the efficacy of analytical assays by negating the need for dedicated laboratories, complex equipment, highly trained personnel, and expensive lab-based infrastructure. These approaches have been applied to a variety of fields from drug discovery for improved molecular assays to biomimetic devices for tissue engineering. ${ }^{12,26}$

In particular, the potential of rapid, quantitative, and sensitive diagnosis has led to many innovative LOC technologies for POC applications. Perhaps the most compelling application of LOC technologies for POC is in the early and accurate detection of infectious agents in developing countries where resources are severely limited. ${ }^{79}$ To be adopted for use in developing countries, the technologies must be extremely inexpensive, robust, scalable, easily adaptable to detecting various infectious agents, sturdy to use under harsh and resource limited environments, and simple to use.

Because POC applications are so cost sensitive (total burdened cost for disposable devices need to be below $\$ 5$ in the developed country and close to pennies in developing countries) effective technologies that can be 
developed with this constraint hold much potential to be leveraged for other applications as well, especially for custom assay development and academic prototyping. ${ }^{20,93}$ The main goal of this review is to therefore provide the reader with a survey of novel fabrication techniques as alternatives to more conventional, expensive, and time intensive traditional approaches. These new approaches offer the promise of more rapid prototyping with less investment in capital equipment as well as greater flexibility in design. Though many of these technologies are still in their infancy, they hold potential to improve upon the resolution, sensitivity, flexibility, and cost-savings over more traditional approaches. Here we focus our review on promising low-cost fabrication techniques for POC applications. We seek to find inspiration here from researchers focused on POC applications that have developed novel low-cost fabrication technologies that obviate many of the legacy micro and nanofabrication processes largely inherited from the semiconductor industry. We start with a brief review of the traditional fabrication processes. Then describe how POC researchers have developed ways to leverage extremely low-cost substrates, and then pattern both structural and biological materials at high resolution in unprecedented ways. The structural material can be patterned for microfluidic channels or for integrated nanostructures for enhanced assay sensitivity. Novel approaches to pattern biological materials are then reviewed.

\section{CONVENTIONAL MICROFABRICATION TECHNIQUES}

The ability to pattern structural and biological materials at high resolution offers unprecedented sensitivity and insight, enabling many recent biomedical investigations. $^{21,33,34,71,86}$ Traditional 'top down' silicon and glass microfabrication techniques-including photolithography, thin film deposition, etching, and bonding - have been leveraged to create complex LOC for applications from immunoassays to PCR reactions to single cell analysis. ${ }^{10,14,47,50,51,56,88,91,97}$ However, largely inherited from the semiconductor industry, these fabrication processes are inherently limited in resolution and planarity by the tooling sources.

In photolithography, patterns are created by UV irradiation of photosensitive polymers for selective cross-linking thus generating the desired topography. ${ }^{72}$ Film deposition involves the formation of thin films on the surface of a substrate and have been used for a variety of purposes in microstructure fabrication. ${ }^{85}$ This has been accomplished by utilizing various chemically and physically driven processes such as chemical vapor deposition, plating, chemical solution deposition, physical vapor deposition, and molecular beam epitaxy. ${ }^{11,25,44,57,85}$ Etching is used to selectively remove or create features on materials and has been accomplished by using physical or chemical methods. ${ }^{9}$ Etching has been performed using either wet methods such as liquid chemicals or dry etching such as gasphase chemistry. ${ }^{44,85}$ Bonding is a method to adhere substrates together to form a hermetic seal. ${ }^{9}$ Depending on the material of interest, different forms of bonding can be applied such as anodic, fusion, thermocompression, or adhesive bonding to obtain desired structures. $^{9,85}$ Most of these processes require legacy capital equipment from the semiconductor industry typically housed in a cleanroom that is extremely costly to run.

In 1998, Whitesides' introduction of PDMS revolutionized the microfluidics field and accelerated its progress by allowing rapid prototyping via soft lithography. ${ }^{16,65}$ Soft lithography utilizes molding or stamping techniques to create micro or nanofeatures from the elastomer, poly(dimethylsiloxane) (PDMS). ${ }^{9}$ While this catalyzed academic progress and has since become the workhorse of microfabrication due to its desirable optical properties (transparency from 240 to $1100 \mathrm{~nm}$ ), flexibility, gas-permeability (for cell based assays), cost-effectiveness, and high patterning fidelity, inherent material properties of this polymer present significant limitations especially for POC applications. ${ }^{68}$ The disadvantages of PDMS include its hydrophobicity, propensity for protein absorption, difficulties in scaling up for mass production, and possible contamination of cyclic silicone monomer derivatives. $^{35,68}$ Due to its high flexibility, channels fabricated using PDMS tend to expand and contract with different pressures. ${ }^{16}$ Although this property benefits some applications, its elastomeric properties could be detrimental to others. These limitations have prevented the industrial use of PDMS for applications in drug discovery and other sensitive assays. ${ }^{68}$ Thus, PDMS has been mainly adopted within academic prototyping and limited to certain applications. ${ }^{38}$ Finally, soft lithography relies on starting with the patterned silicon wafer and therefore still relies on standard photolithography processing.

Thermoplastic materials including polymethylmethacrylate (PMMA), polycarbonate, polystyrene (PS) and cyclic olefin polymers are commonly used for research laboratory disposables including microtiter plates and petri dishes. These plastics are attractive because they are inexpensive, robust, possess good optical properties, and their surfaces can be easily modified. ${ }^{33}$ Patterning large scale features into these plastics are extremely affordable as they are compatible with roll to roll processing. 
However, current manufacturing of finer (micro or smaller) features directly into hard plastics typically necessitates expensive capital equipment and extensive processing steps. In hot embossing, a silicon master must first be created with the features defined photolithographically and subsequently chemically etched or used to create serial nickel electroforms. Each step of the mold making process requires significant processing time and large dedicated fabrication equipment. Once the mold is made, the imprint can be performed with high fidelity using an automated hot embosser. The hot embossing machine heats up the plastic under high pressure conditions when in contact with the mold for pattern transfer into the plastic. ${ }^{39}$ Similarly, injection molding starts with the silicon master and a nickel electroform. The nickel electroform is then mounted into a mold insert where the polymer solution is injected. ${ }^{40}$ Creating a precise stamp with microfeatures is time and labor intensive. ${ }^{48,75,78,89}$ Furthermore, the physical limitations of hot embossing systems and properties of the plastic material limit the thickness of the embossed plastic patterns. ${ }^{36}$ While such approaches could become cost-effective for extremely high-volume production, for prototyping - as typical in academic research as well as in small companies - they are prohibitively expensive both in terms of setup costs as well as the cost per chip. For many researchers, such expensive specialized equipment, a clean room, and costly consumables are not adaptable at their universities or companies. ${ }^{85,87}$

\section{ALTERNATIVE LOW-COST SUBSTRATES AND RAPID PATTERNING OF MICROFLUIDIC CHANNELS}

In addition to the expensive capital costs associated with traditional microfabrication approaches, another notable disadvantage is the required fabrication time. Methods such as soft lithography and hot embossing require first patterning silicon wafers to serve as 'master's which requires many hours to several days. Therefore, multiple design iterations, inevitable in prototyping, becomes extremely slow and costly. Moreover, the features are limited to predominantly planar features. Novel substrates and patterning techniques developed for POC illuminate exciting possibilities for alternative approaches.

The enzyme-linked immunosorbent assay (ELISA) for antibody detection has remained the gold standard method of antibody detection since Engvall and Perlman introduced it in the $1970 \mathrm{~s} .{ }^{27}$ A typical ELISA is performed in a 96 well microtiter plate in which an antibody is bound to a solid material such as a plastic bead or planar substrate in order to capture antigens from the sample. ${ }^{49}$ Following this, a second enzymelabeled detection antibody is added. After multiple washing steps to remove unreacted reagents, a fluorescence microscope is used to image the substrate. ${ }^{18,92}$ ELISA requires long assay times, use of expensive reagents and equipment, multiple washing steps and a trained clinician. ${ }^{92}$ While the cost for an ELISA testing varies from $\$ 2$ to $3 /$ sample, the infrastructure required to run an ELISA can amount to almost $\$ 30,000$.,

Paper serves as an excellent material to fabricate bioassays because it has the ability to wick fluids, is inexpensive, biodegradable, readily available, and easy to functionalize. ${ }^{18,24}$ Recently, Cheng et al. ${ }^{18}$ developed an ELISA assay made out of paper (p-ELISA). Here, using paper rather than the conventional plastic wells, they were able to lower the cost, time, and volume of reagent required to perform ELISA. In their approach to fabricate a p-ELISA, the group applied SU-8 photoresist onto paper and followed conventional photolithography techniques for patterning the array. In their device, the optimal size of the detection zones was $5 \mathrm{~mm}$ in diameter, and to completely wet this area, only $3 \mu \mathrm{L}$ fluid was required. Furthermore, when the size of the detection zone was reduced to a diameter comparable to the 384-well plate format, the volume required to completely wet the detection area was reduced to $0.5 \mu \mathrm{L}$. Within an hour, the entire analysis was completed in contrast with a traditional ELISA, in which the incubation steps can take up to an hour. The efficiency and accuracy of the device were evaluated using a colorimetric bioassay. Rabbit IgG at various concentrations were immobilized in detection areas, blocked, followed by incubating with a solution of alkaline phosphatase (ALP) conjugated anti-rabbit goat IgG. Currently, however, their limit of detection is approximately tenfold higher than the traditional ELISA, but the ability to mass produce these devices is an attractive advantage. ${ }^{18}$

Martinez et al. also pioneered the fabrication of micro-paper-based analytical devices ( $\mu$ PADs) by patterning SU-8 photoresist onto chromatography paper using traditional photolithography techniques (Fig. 1). ${ }^{24,62}$ In order to increase the hydrophilicity of the paper which may have been affected by residual photoresist, the surface was treated with oxygen plasma. To demonstrate the functionality of their device, glucose and protein were spotted in the test zones created (Fig. 1). For the glucose assay, colorless iodine was oxidized to brownish iodine via the enzymatic reaction of glucose oxidase with horseradish peroxidase. Within 10 min after the glucose was spotted, the color was fully developed and results were obtained. Furthermore, they were able to run 20 different samples within $8 \mathrm{~min}$ and observe the results within 20 min. $^{62}$ The sensitivity detection limit of $5 \mathrm{mM}$ was 
comparable to commercially available dipsticks for glucose detection.

Bruzewicz et al. subsequently presented the idea of generating hydrophilic channels by using an $x-y$ desktop plotter to print PDMS onto filter paper. ${ }^{13,24}$ Unlike photoresist or PMMA, PDMS is an elastomer that is relatively more flexible so channels could easily be folded or bent without destroying the channel. ${ }^{13}$ Moreover, the PDMS solution was able to penetrate the paper generating hydrophobic barriers. Carrilho et al. ${ }^{15}$ furthered improved the idea of fabricating hydrophilic channels in their $\mu$ PADs by printing walls of hydrophobic wax on paper in order to fabricate microfluidic channels which were approximately $500 \mu \mathrm{M}$ wide that have hydrophobic barrier. Utilizing this process, they were able to create $\mu$ PADS that were made within 5 min. ${ }^{15}$ In this process, a wax ink printer was utilized to print a fluidic layout onto paper. ${ }^{15,24}$ Following this, the wax was heated which led to lateral diffusion and vertical spreading along the paper forming the hydrophobic walls. While the resolution of the channel features made through conventional photolithography is better for the purpose of fabricating $\mu$ PADs, wax printing would work sufficiently. ${ }^{15}$ In an independent work, Lu et al. ${ }^{58,59}$ also demonstrated that wax can be utilized for patterning microstructures on filter paper and nitrocellulose membrane within $10 \mathrm{~min}$. Three different modes of printing wax onto filter paper were applied including using a wax pen, an inkjet printer followed by a wax pen, and a wax

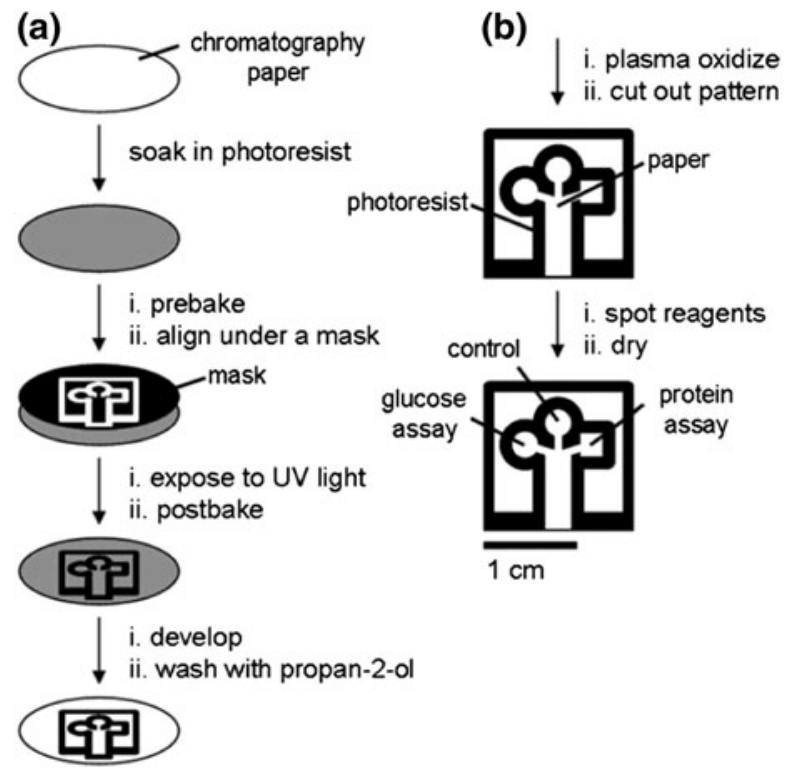

FIGURE 1. Schematic of the paper patterning method. (a) SU8 photoresist was patterned onto paper; (b) The millimetersized channels were then modified for biological assay. Figure reproduced with permission from Martinez et al. ${ }^{62}$ Copyright 2007 Wiley-VCH Verlag GmbH \& Co. KGaA. printer. Wax serves as a promising alternative for patterning on paper rather than using SU-8 and PDMS because of its advantages of being inexpensive, user friendly, and environmentally friendly.

Martinez et al. showed that three-dimensional (3D) microfluidic devices could be easily fabricated by assembling alternate layers of SU-8 patterned paper with double-sided adhesive tape. ${ }^{24,64}$ By patterning SU-8 photoresist onto the paper, hydrophilic channels could be easily created and fluids were able to move vertically through the layers by laser cutting holes through the tape (Fig. 2). To improve the wicking properties between the adjacent layers of papers, cellulose powder was added between the gaps created by the thickness of the tape. The device has four channels which intersected with each other multiple times in a basket weave pattern and were approximately $800 \mu \mathrm{M}$ wide and $5 \mathrm{~cm}$ long. These devices are relatively easy to make and inexpensive, only costing $\$ 0.03$. To demonstrate the functionality of the devices, glucose and protein assays were performed within the device. More recently Martinez et al $^{63}$ fabricated 3D $\mu$ PADs that enabled the user to design the structure of the channels, control the fluid flow, and control the device functions, post-fabrication. An "on" button was fabricated by utilizing the gap distance created by the tape between the adjacent layers of papers. Unless a hydrophilic material such as cellulose powder was used to fill the gap, fluid would not flow. Thus, by using a standard narrow object such as a ball point pen, the gap could be closed and the devices could be manually programmed by the user.

Thread was used as the simplest and fastest method for fabrication of low-cost diagnostic devices. In this method cotton was used as the thread of choice because it is low-cost, accessible, durable, has a high aspect ratio, and requires no external power to move fluids along the thread. ${ }^{54,77}$ In order to have the cotton thread wick fluids well, Li et al. plasma treated the cotton thread before sewing the threads onto a translucent polymer film fabricating 3D-like microfluidic devices. ${ }^{54,77} \mathrm{Li}$ et al. demonstrated that there were able to promote mixing of fluids in desired locations by simply twisting two hydrophilic threads. Reches et al. used an alternative type of cotton, mercernized cotton, which is hydrophilic to fabricate their diagnostic assays, eliminating the need for plasma treatment. Similar to $\mathrm{Li}$ et al., they also manually sewed the thread into various substrates such as bandages and plastic. However, they also explored two other designs where the thread was sandwiched between two pieces of scotch tape. By sandwiching the thread, they were able to rapidly and effectively wick the fluids. As a proof of concept, they used their thread-based microfluidic devices to perform qualitative colorimetric 

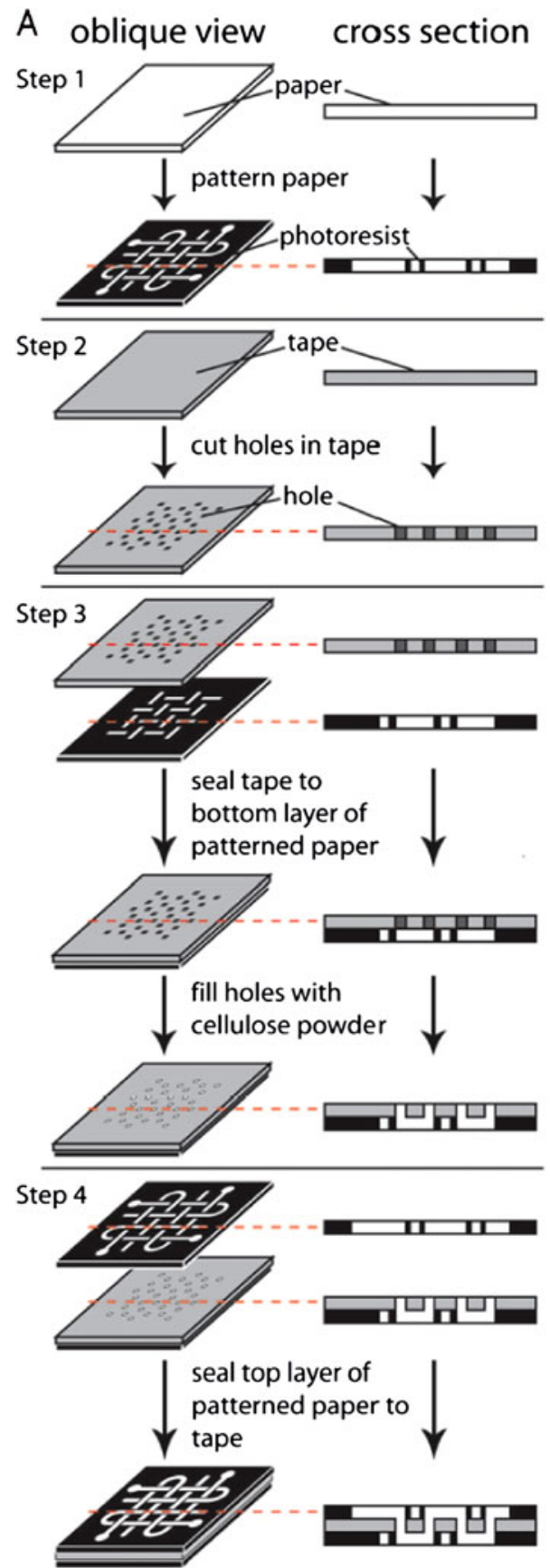

FIGURE 2. Overview of the $\mu$ PADs fabrication procedure. Adapted with permission from Martinez et al. ${ }^{64}$ Copyright (2008) National Academy of Sciences, U.S.A.

assays for nitrite, protein, ketones, glucose, and alkaline phosphatase. ${ }^{77}$

Alternative fabrication approaches have also been developed to make plastic substrates an affordable solution. Grimes et al. $^{36}$ introduced an inexpensive alternative for fabrication of microfluidic devices by utilizing thermoplastics such as polystyrene (PS). More recently, Nguyen et $a .^{71}$ leveraged polyolefin (PO) film. PO which has been commonly used for the manufacturing of shrink wrap films, possesses excellent shape memory properties that can be recovered through the application of heat. ${ }^{71}$ Other attractive properties of the PO films are optical properties such as transmission over a large range of wavelengths in addition to low autofluorescence. ${ }^{71}$ It was also shown that by leveraging the shrinkage property of the PO sheets, high resolution microstructures can be generated obviating the need for photolithography and advanced tooling (Fig. 3). To demonstrate the applicability of this polymer, fluorescently tagged polyclonal antibodies proteins were stamped onto the PO surface via microcontact printing $(\mu \mathrm{CP})$. After this, the substrate was heated and an increase in fluorescence was demonstrated indicating an increase in concentration due to the $95 \%$ shrink-induced decrease in surface area.

McKenzie et al. ${ }^{66}$ fabricated a microfluidic device that could be used for rapid removal or separation of immunoglobin, IgG, from human plasma. In their technique, plastic laminates (adhesive backed Mylar and poly(methyl methacrylate) PMMA) were cut with a $25 \mathrm{~W} \mathrm{CO}$ laser to create microfluidic cards. In between the polymers layers, porous hydrophobic membranes were sandwiched to act as a fluid stop and vent for air bubbles. A mixer was created in which mixing occurred by air bubbles created by a pneumatic source. Within the mixing chamber, protein $\mathrm{G}$ immobilized beads in a viscous sucrose solution were dried for approximately 24-48 h. Protein G, a bacterial cell wall protein, was chosen for it specificity for binding to IgG. In addition to a mixer, a polyester mesh net was used as a filter for removing the beads. To test the functionality of their device, buffer spiked with FITC labeled human IgG or diluted human plasma samples were loaded into the inlet port of the microfluidic card device and were sent through the bead filter and held within the fluid reservoir. With air pressure, the sample was pushed into the mixing chamber and mixed with rehydrated beads for $5 \mathrm{~min}$ before pulling the sample out of the chamber. Finally, the sample was re-sent through the bead filter to remove beads and pipetted off the card for analysis. The results demonstrated that after $5 \mathrm{~min}$ of processing, approximately $66-77 \%$ of $\mathrm{IgG}$ was removed from the human plasma samples.

Recently, Yuen and Goral ${ }^{96}$ utilized a desktop digital craft cutter to fabricate flexible microfluidic devices within minutes. Using double-sided pressure sensitive adhesive (PSA) tape and laser printer transparency film, they were able to fabricate 3D microfluidic channels with $200 \mu \mathrm{M}$ thin microchannels. More recently, Nath et al. ${ }^{70}$ used PSA tape to fabricate various microfluidic components such as a micro-mixer, dielectrophoresis, and a high temperature bioreactor that could be integrated into lab on chip devices. Chen et al. and 


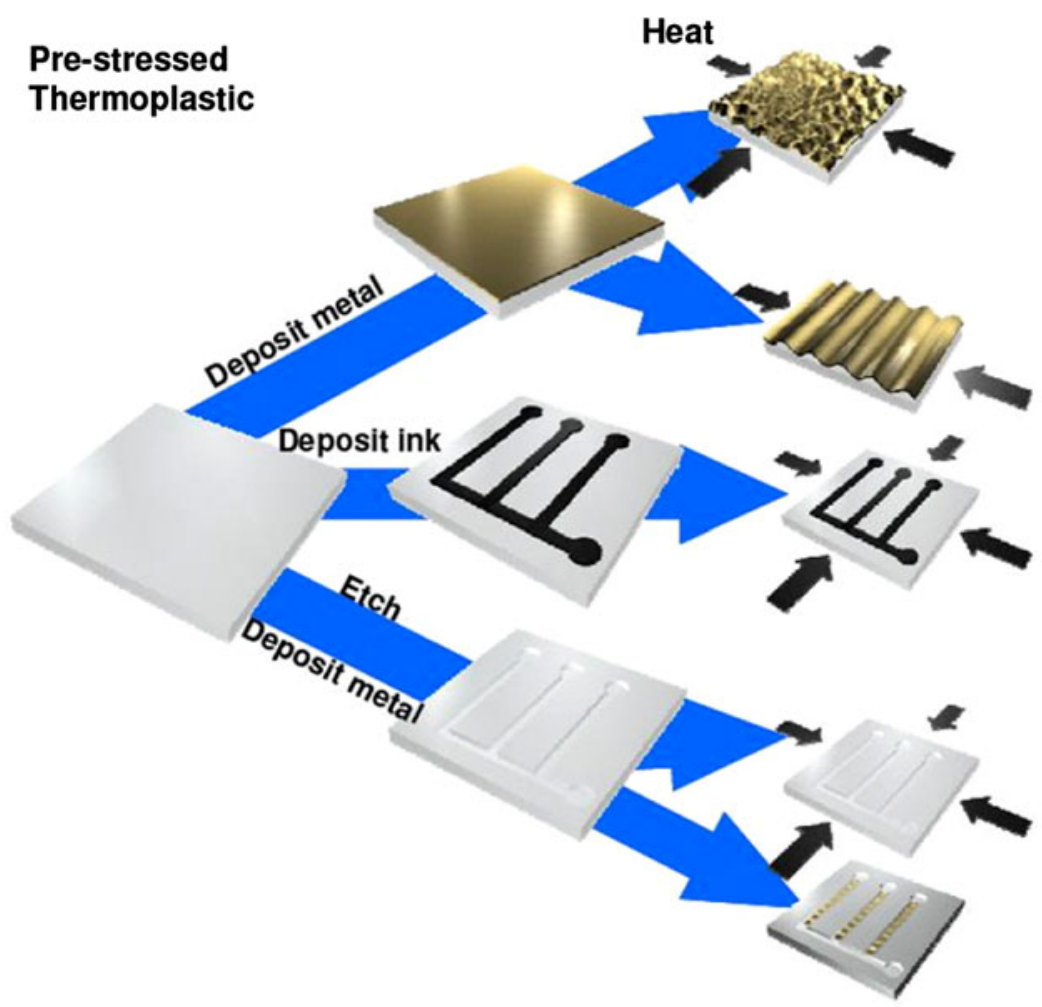

Nanostructures

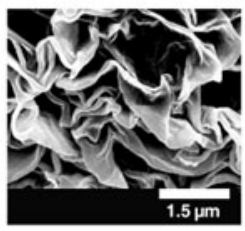

Microfluidic Chips
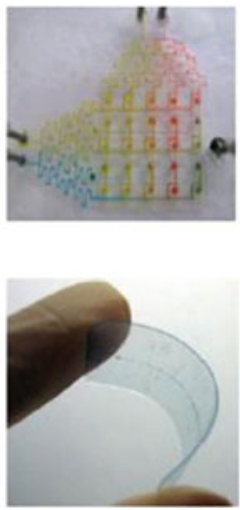

FIGURE 3. Ultra-rapid, low-cost manufacturing process of nano/micro-systems.

Taylor et al. also fabricated complete 3D microfluidic chips by stacking layers of PS or PO and heating them stacking the thermoplastic layers (Fig. 3). ${ }^{16,84}$ The functionality of the chip was assessed by performing a sandwich immunoassay within the device and the results showed an increase in fluorescence intensity postshrinkage. Sollier et al. utilized "Print-n-Shrink" technology to fabricate their microfluidic devices. In their method, a screen printer was used to print dielectric ink onto a shrinkable polystyrene sheet and then heated to induce shrinkage. ${ }^{80}$ To test the functionality of their microfluidic chips, proteins were spotted on the polystyrene film, shrunk and imaged using a fluorescence microscope.

Sudarsan et al. utilized PS-based thermoplastic elastomer gels to create viscoelastic three-dimensional (3D) microfluidic chips using a molding approach. ${ }^{83}$ In their approach gels were synthesized by mixing cheap polystyrene-polyethylene-polybutylene (SEBS) triblock copolymers in mineral oil and leaving the mixture of resin and oil overnight under vacuum. In order to allow the resin and oil to mix with each other and remove any remaining air pockets, the mixture was placed under vacuum and heated to $120-170{ }^{\circ} \mathrm{C}$ for approximately $4 \mathrm{~h}$. Since, the butylene and ethylene midblocks are selectively soluble in mineral oil while the styrene is insoluble, the strong repulsion between the blocks forced microphase separation and the styrene end blocks self-assembled into localized nanodomains. The viscoelastic gel network was formed when the soluble midblocks permeated into the oil generating arrays of bridges and loops and the polystyrene domains served as cross-link junctions. ${ }^{83}$ After the mixture was left to cool to room temperature, the solid gel was sliced into small pieces and placed on top a PC board master mold preheated to $120^{\circ} \mathrm{C}$. Once the elastomer was malleable, gentle pressure was applied manually to ensure complete contact with the mold. Unlike PDMS, the same elastomer slab could be used to make different impressions with other masters that have distinct features. To test the biocompatibility of the substrate, the inner portion of polymerase chain reaction (PCR) tubes were coated with $23 \mathrm{wt} \%$ elastomer gel and standard DNA amplification using the coated PCR tubes was performed in a thermocycler for $1 \mathrm{~h}$. Standard gel electrophoresis was run on the samples and the results demonstrated that the elastomeric gel was biocompatible with DNA and standard biochemical reagents.

Wang et al. introduced a novel method, lab on print (LOP), to fabricate 3D microfluidics in less than an hour. ${ }^{29,86}$ In their method, graphic design software was utilized to fabricate micro-scale patterns and then printed using a solid ink printer to deposit wax on both 
sides of a $25-125 \mu \mathrm{m}$ thick polyimide film. Isotropic etching into the polyimide substrate was performed using a $\mathrm{KOH}$-based wet etching bath. In this solution, only the polyimide substrate was able to be etched while the wax acted as an etching barrier. Finally, the polyimide film was folded and the wax layers were thermally bonded onto each other. To test the functionality of their device, a microfluidic generator was
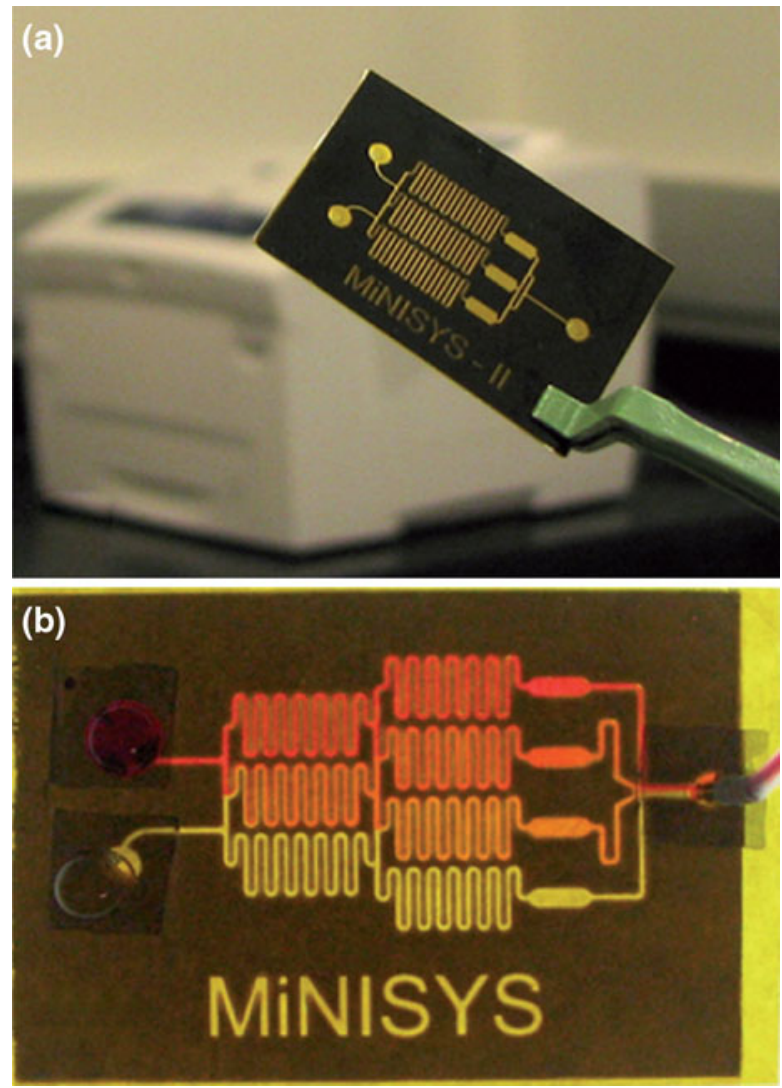

FIGURE 4. Microfluidic gradient generator fabricated using LOP process. (a) Substrate composed of polyimide (yellow) is patterned with wax (black); (b) Chemical gradient of red dye buffered with DI water. Figure reprinted with permission from $^{86}$-reproduced by permission of Royal Society of Chemistry. fabricated with their technique (Fig. 4a). Red color dye and de-ionized water (DI) water were loaded into two separate inlets to essentially create mixing chemical gradients and the outlet was connected to a vacuum pump. The pressure difference between the inlet and outlet pushed the red dye and DI water into the microfluidic device and generated a gradient downward due to diffusive mixing (Fig. 4b).

Another out of the cleanroom microfabrication alternative for 3D microfluidic structures was also introduced by Zhao et al. ${ }^{98}$ known as Direct Projection on Dry-film Photoresist (DP). A digital projector was utilized to generate masks and operate as a photo exposure system. An easy processing dry-film photoresist was used for microfabrication and 3D microfluidic structures were fabricated within an hour. With this technique, they were able to achieve a resolution of $10 \mu \mathrm{m}$ using thick dry-film resist.

Recently, in a novel process developed by Cheung et al. ${ }^{19}$ it was shown that $3 \mathrm{D}$ structures can be fabricated within microfluidic chips utilizing photocurable acrylate-based polymers and standard fluorescent microscopy. In this approach, by flowing the photocurable polymers through a microfluidic device composed of PDMS chambers sandwiched between glass slides, and exposing the chambers to fluorescent light they were able to selectively generate $3 \mathrm{D}$ structures within the device. It was also shown, that the height of these structures can be tuned by varying the height of the microfluidic chambers. Notably, due to the low viscosity, it was possible to flow the polymers through the chambers using standard pressure driven flow. Thus, by selectively scanning the light beam within the desired region, it was demonstrated that controlled patterns of $3 \mathrm{D}$ polymers can be generated, while uncured regions can easily be washed away. In this study it was shown that up to 24 different photocurable polymers can be selectively polymerized within minutes compared to standard lithographic methods which required hours. A summary of the low cost substrates described in this section is presented in Table 1.

TABLE 1. Low-cost substrates.

\begin{tabular}{|c|c|c|c|}
\hline Low-cost fabrication material & Advantages & Applications & Types \\
\hline Paper-based ${ }^{13,15,18,58,59,62,64,70}$ & $\begin{array}{l}\text { Biodegradable, accessible, ease } \\
\text { of functionalization, hydrophilic }\end{array}$ & ELISA, microfabrication & Cellulose, nitrocellulose \\
\hline Thread-based ${ }^{54,77}$ & $\begin{array}{l}\text { Accessible, rapid fabrication, high } \\
\text { aspect ratio, hydrophilic, ease } \\
\text { of functionalization }\end{array}$ & $\begin{array}{l}\text { Colorimetric assays, } \\
\text { microfabrication }\end{array}$ & Cotton \\
\hline Thermoplastics ${ }^{36,70,71,80,84,96}$ & $\begin{array}{l}\text { Desirable optical properties, durable, } \\
\text { inexpensive, ease of surface } \\
\text { modifications }\end{array}$ & $\begin{array}{l}\text { Microfabrication, immuno } \\
\text { assays }\end{array}$ & $\begin{array}{l}\text { PS, PO, PMMA, SEBS, } \\
\text { COC, polycarbonate, } \\
\text { PSA, PI }\end{array}$ \\
\hline Photocurable polymers ${ }^{86,98}$ & $\begin{array}{l}\text { Rapid fabrication, high aspect ratio, } \\
\text { low viscosity, high controllability }\end{array}$ & $\begin{array}{l}\text { Microfabrication/3D } \\
\text { microfabrication }\end{array}$ & $\begin{array}{l}\text { DP, acrylate-based } \\
\text { photoresist }\end{array}$ \\
\hline
\end{tabular}




\section{INTEGRATION OF NANOSTRUCTURES FOR ENHANCED SENSITIVITY}

Nanostructures integrated into the substrates can offer increased assay sensitivity. Wu et al ${ }^{90}$ fabricated nanopores as a mode for DNA detection in a relatively inexpensive thermoplastic by using a laser as their heat source. In this method, a $200 \mu \mathrm{M}$ diameter pore was mechanically punctured out in the center of a 700-900 $\mu \mathrm{M}$ thick membrane. An argon ion laser was used to supply a continuous-wave laser beam to the punctured area and within minutes the hole reduced by almost 1000 -fold to a few hundred nanometers. To characterize and demonstrate the functionality of their nanopores, double-stranded $\lambda$-DNA was added to their device which was submerged in ionic solution and negative potential was applied to force the DNA molecules to move through the pores. Based on the results obtained from a current readout and PCR, they were able to detect DNA.

Molecular based plasmonic sensing involves the manipulation of light in the sub wavelength regime. Nanostructured metals with free electrons can produce surface plasmon oscillations resulting in strong surface-bound electromagnetic fields when resonantly excited using visible light; these resulting surface plasmon resonance (SPR) fields can be used to detect biomolecules in a variety of ways. Gao et al. ${ }^{32}$ used angle dependent resonances from plasmonic crystals for biosensing applications. Soft interference lithography and PEEL (phase-shifting photolithography, etching, electron-beam deposition, and lift-off of the film) were used to fabricate a silicon template of a $2 \mathrm{D}$ square array of nanopyramidal pits. ${ }^{32,42}$ PDMS was used to capture the patterned features on the silicon mold and transfer the features to UV-curable polyurethane (PU). To generate the 2D plasmonic crystals, $150 \mathrm{~nm}$ gold was electron-beam evaporated (E-beam) onto the molded PU substrates. For testing the functionality of their device, the molded gold plasmonic crystals were functionalized with a self-assembled monolayer, SAM. By simply tuning the angle of incident light, they were able to observe the plasmon resonance shift to longer wavelengths as proteins bound to specific receptors on the substrate. Furthermore, by tuning the excitation angle, they were able to increase the signal to noise ratio. More recently, Yang et al. ${ }^{94}$ fabricated 3D nanohole arrays using a similar procedure. Lee et al. used the process of solvent-assisted nanoscale embossing (SANE) to generate plasmonic nanoparticle arrays that could be utilized for fabrication of biosensors in the future. ${ }^{42,52}$ In their method, PDMS was cast against the PU master mold with hexagonal array of posts to generate PDMS molds (Figs. 5a, 5b). The PDMS mold was then wet with a solvent before conformal contact with photoresist on silicon wafer (Fig. 5c). Another approach that was applied to fabricate new arrays with higher densities was performed by first wetting the PDMS mold with photoresist and then using a convection oven to heat the patterned resist with shrink film. Within $40 \mathrm{~min}$, the thermoplastic shrunk by $60 \%$ and this helped with reducing the separation between the features. Furthermore, to increase the spacing between the features, the thermoplastic could also be mechanically stretched. This new master could then be molded again with PDMS and then wet with solvent before having conformal contact with a photoresist coated substrate on a silicon wafer. SANE methods were also applied to fabricate metallic nanoparticle arrays and the spacing between the particles was tuned by varying the shrinking time of the polymer. The ability to control these metallic nanostructures can be utilized for fabrication of plasmonic biosensors.

Stewart et al. created quasi-3D plasmonic crystals for potential use in label-free detection systems. ${ }^{81,95} \mathrm{In}$ their method, soft nanoimprint lithography was utilized to fabricate nanostructured substrates by embossing PDMS that had square arrays of cylindrical features into polyurethane on a glass slide. The polyurethane layer was left to cure by exposure to UV light before the PDMS stamp was carefully removed, leaving behind nanofeatures. Thick gold films were deposited on the patterned substrate by using electronbeam deposition to generate quasi 3D plasmonic crystals or sputtered to create "full" 3D plasmonic crystals. The nanoscale holes produced the SPR effects in the gold film.

Integrating these metal structures into microfluidic devices holds promise for biosensing applications. Hidber et al. first utilized microcontact printing and electroless deposition to fabricate patterned surfaces on biaxially preoriented films of polystyrene. ${ }^{43,99}$ The inherent ability of the thermoplastic to shrink above its glass transition temperature induced the platinum colloids on the thermoplastic to form microfeatures that were pronounced after electroless deposition of copper. Fu et al. ${ }^{30}$ also demonstrated a novel method to fabricate metal nanowrinkles by using a two-step approach and tailored the scale range for the nanometer metal wrinkles by adjusting the thickness of the metal deposited on the polystyrene sheets. In their method, a thin layer of gold was deposited on polystyrene sheets via sputtering after which the substrate was heated to $160{ }^{\circ} \mathrm{C}$ to form metallic wrinkles due to stiffness mismatches (Fig. 3). The wrinkles were also integrated into shrink-induced polymer microfluidics devices. To demonstrate the utility of wrinkles for POC diagnostics, dye molecules were dissolved in polymer solution and spin coated on the gold wrinkles. To 
(a)

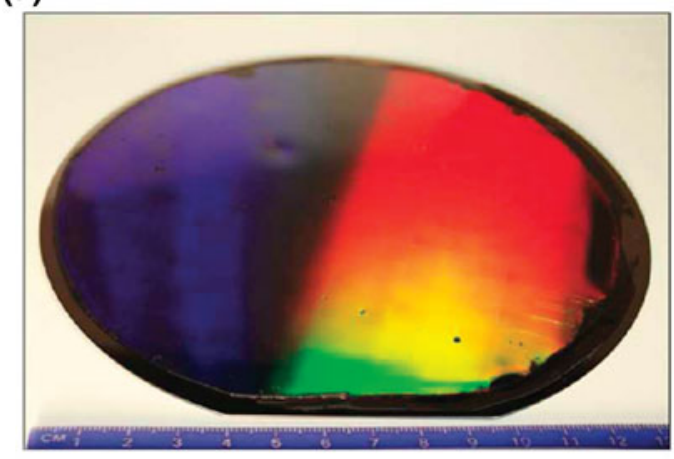

(b)

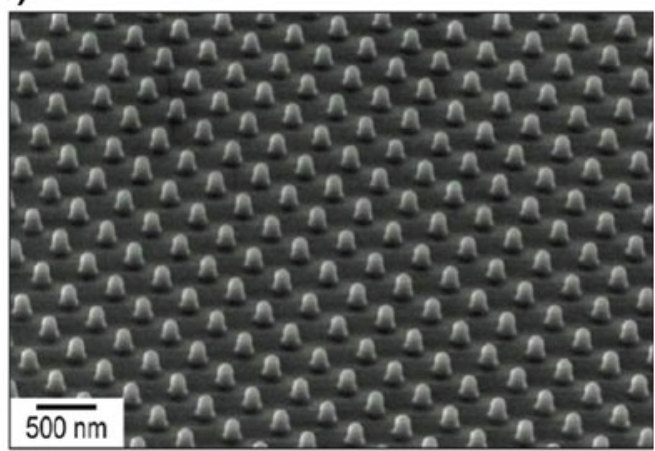

(c)

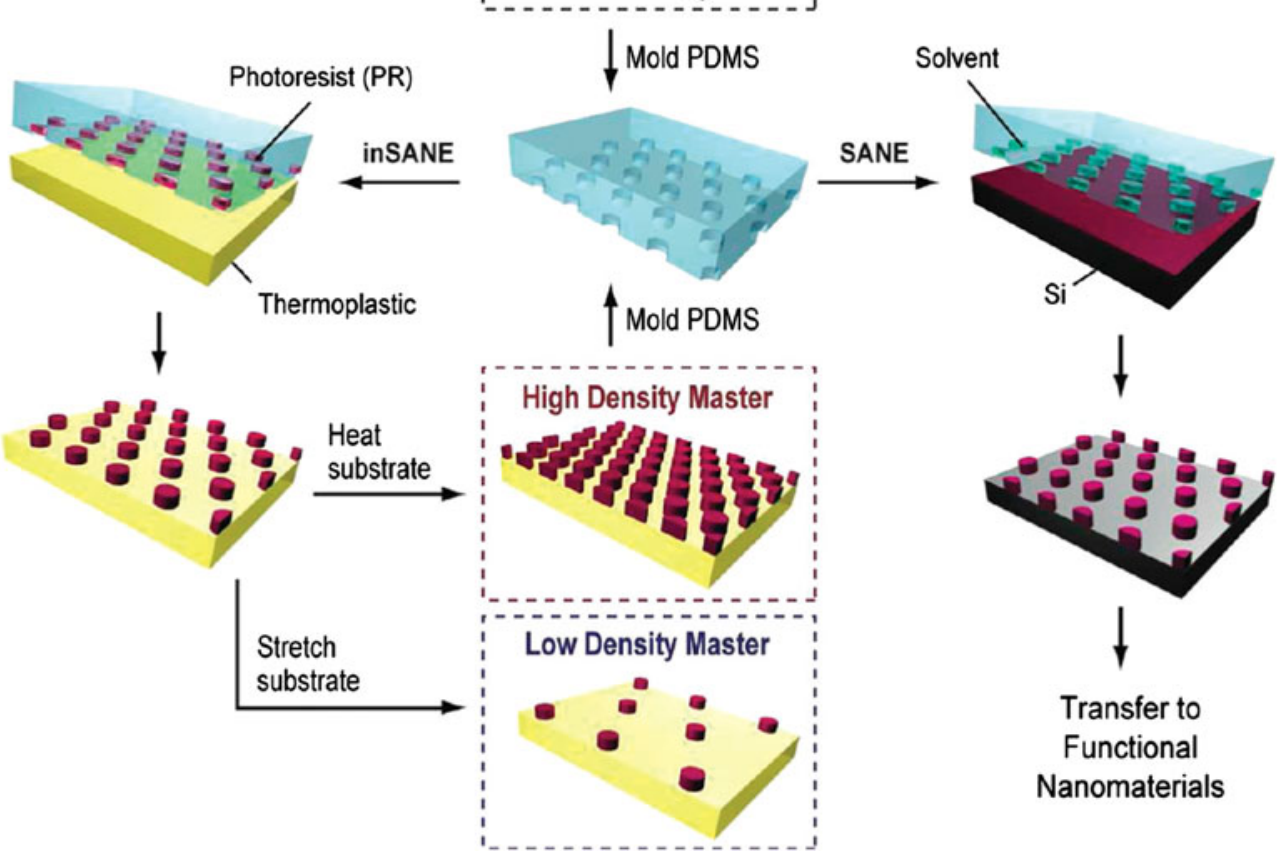

FIGURE 5. Schematic of solvent-assisted nanoscale embossing. (a) Optical micrograph of PU master; (6 in) diameter; (b) SEM images of nano-structures on PU master; (c) Schematic of inverse SANE (inSANE) fabrication procedure for the generation of high and low density nanostructures. Figure reprinted with permission from Lee et al. ${ }^{52}$

decrease the size of the nanostructures and increase the density of the hot spots, Fu et al. then developed a way to crack open the petals and demonstrated a 4000 -fold increase in the fluorescence enhancement due to SPR effects. $^{31}$

Sia et al. introduced a portable and cost-effective (POCKET) immunoassay that was used to quantify anti-HIV-1 antibodies in human patient sera. ${ }^{79}$ In their device, antibodies conjugated to gold colloids catalyzed the reduction of silver ions to silver atoms. The detection was performed by using a InGaAlP red semiconductor laser diode $(654 \mathrm{~nm})$, and optical integrated circuit that acted as a photodetector. The opacity of the silver film was correlated to the concentration of analyte. Furthermore, incubation times within the microfluidic device were only $10 \mathrm{~min}$. Recently, Luo et al. ${ }^{60}$ integrated a PDMS microfluidic device with surface plasmon resonance imaging to fabricate an immunoassay that could detect at the subnanomolar level. Liu et al. ${ }^{55}$ designed a nanoplasmonic molecular ruler in which double-stranded DNA was attached to a gold nanoparticle and a shift in the plasmon resonance wavelength corresponded to the length of the DNA. A summary of the biosensing 
TABLE 2. Micro and nanofabricated substrates with applications in biosensing.

\begin{tabular}{|c|c|c|c|}
\hline Description & Advantages & Required instrumentation/consumables & Applications \\
\hline Nanopores in thermoplastic ${ }^{90}$ & $\begin{array}{l}\text { Lithography-free, ability to tune } \\
\text { size of nanopores }\end{array}$ & Argon ion laser, thermoplastic & DNA sensor \\
\hline Plasmonic crystals ${ }^{32}$ & $\begin{array}{l}\text { Label-free, large area plasmonic } \\
\text { sensing substrates, does not } \\
\text { require electron-beam lithography } \\
\text { or focused ion beam milling }\end{array}$ & $\begin{array}{l}\text { Polyurethane, E-beam evaporation, } \\
\text { PDMS, Si, gold }\end{array}$ & $\begin{array}{l}\text { Protein sensor, } \\
\text { drug screening }\end{array}$ \\
\hline SANE $^{52}$ & $\begin{array}{l}\text { Ability to tune separation between } \\
\text { patterns, uniform patterns }\end{array}$ & $\begin{array}{l}\text { Shrink film, PDMS, Si, oven, } \\
\text { photo-resist, ethanol, gold }\end{array}$ & Biosensor \\
\hline Quasi-3D plasmonic crystals ${ }^{81}$ & Label-free, uniform crystals & $\begin{array}{l}\text { PDMS, polyurethane, E-beam, } \\
\text { UV light, gold }\end{array}$ & Biosensor \\
\hline $\begin{array}{l}\text { Patterned catalyst on } \\
\text { polystyrene }\end{array}$ & $\begin{array}{l}\text { Photolithography-free, tunable } \\
\text { patterns }\end{array}$ & $\begin{array}{l}\text { Palladium colloids, polystyrene } \\
\text { sheets, PDMS, oven, copper } \\
\text { plating bath }\end{array}$ & Biosensor \\
\hline Metal nanowrinkles ${ }^{30,31}$ & Tunable nanowrinkles & $\begin{array}{l}\text { Sputter coater, polystyrene, oven, } \\
\text { gold, silver }\end{array}$ & $\begin{array}{l}\text { Immunoassay, } \\
\text { DNA sensors }\end{array}$ \\
\hline POCKET immunoassay ${ }^{79}$ & $\begin{array}{l}\text { High sensitivity (LOD: } 163 \mathrm{pM} \text { ), } \\
\text { integrated into microfluidic device, } \\
\text { electricity-free }\end{array}$ & $\begin{array}{l}\text { InGaA1P red semiconductor laser } \\
\text { diode, gold, silver }\end{array}$ & Immunoassay \\
\hline Gold nanoparticles ${ }^{60}$ & $\begin{array}{l}\text { Integrated into microfluidic device, } \\
\text { sensitivity detection limit: } 38 \mathrm{pM}\end{array}$ & $\begin{array}{l}\text { PDMS, gold nanoparticles, oven, } \\
\text { photoresist, silicon, printer }\end{array}$ & Immunoassay \\
\hline Nanoplasmonic ruler ${ }^{55}$ & $\begin{array}{l}\text { Label-free, ability to study kinetics } \\
\text { of nuclease enzymatic reactions }\end{array}$ & $\begin{array}{l}\text { Metal nanoparticles, phosphine } \\
\text { moiety, ultracentrifuge, } \\
\text { scattering spectroscopy }\end{array}$ & DNA footprinting \\
\hline
\end{tabular}

substrates described in this section is presented in Table 2.

\section{LOW-COST MOLECULAR PATTERNING TECHNIQUES}

The ability to pattern biomolecules onto surfaces is critical for biological research such as proteomics, genomics, fabrication of biosensors, and engineering of tissue scaffolds. ${ }^{6,22}$ Recently, several research groups have proposed novel methods of patterning or dispensing biomolecules onto surface.

Huo et $a l^{45}$ described polymer pen lithography (PPL), an innovative method to deposit inks in a "direct write" manner. In this method, a silicon master is made from established photolithography procedures which is used to fabricate a polymer pen array containing thousands of pyramid-shaped tips. ${ }^{45} \mathrm{~A}$ year later, Zheng et al. ${ }^{100}$ proposed a rapid method that involved using PPL for inking nanoscale probes with various types of proteins that did not result in crosscontamination (Fig. 6a). A $5 \times 5$ protein dot array was made by using each pen in the array (Fig. 6d). By simply varying the tip-substrate contact time and contact force, they could control the feature size from the nano- to the micro-scale.

Baserga et ll $^{8}$ utilized dip pen lithography (DPN) and template stripping (TS) techniques to fabricate a nanoarray that could be used for label-free DNA detection. In DPN, the biomolecules are coated on the AFM tip and deposited onto the substrate, enabling submicron scale molecular patterns. ${ }^{76}$ To fabricate their substrate, they combined techniques of TS and evaporation of metals through a grid mask. The principle of the TS technique involves depositing metal onto a freshly cleaved mica surface using physical vapor deposition (PVD) methods. ${ }^{8,41}$ The freshly coated metal surface was glued onto a silicon wafer and the mica layer was stripped by chemical or mechanical means. Once the mica layer was stripped, the metal film deposited initially on the mica surface was almost as flat as the mica. The flatness of the substrate enabled the atomic force microscope (AFM) and scanning tunneling microscope (STM) to characterize the biomolecules on the substrate that were deposited using DPN. Lee et al. ${ }^{53}$ showed that TS of gold films could also be performed in ultrahigh vacuum to generate clean, flat surfaces that could be used as a substrate for highly ordered self-assembled monolayer (SAM) formation.

In diagnostic applications such as lateral flow strips, use of aerosol deposition and contact striping methods for the patterning of biomolecules onto cellulose-based substrates requires the use of expensive commercial equipments which may limit its application in POC diagnostics. ${ }^{37,61}$ Nash et al. ${ }^{69}$ demonstrated that the fabrication of lateral flow strips can easily be fabricated by utilizing the common lab equipment: a syringe pump. In this approach, anti-streptavidin antibodies 
(a)

Inkjet-print multiple protein inks
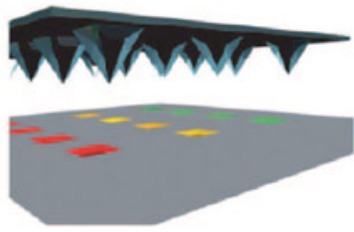

(b)
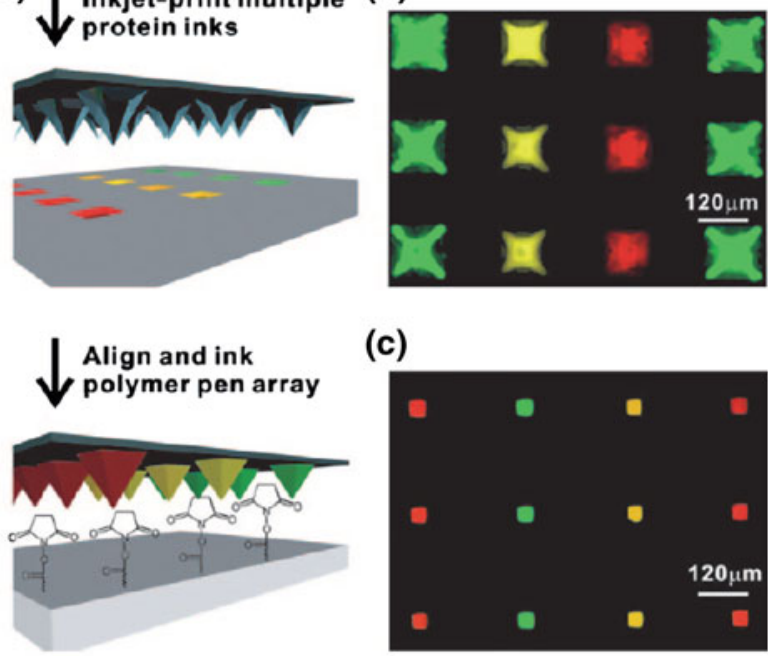

(c)

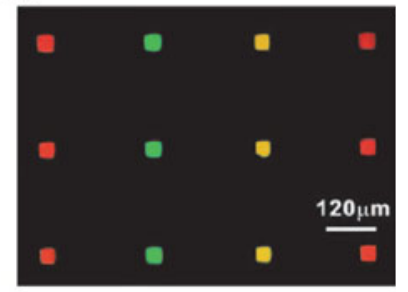

(d)

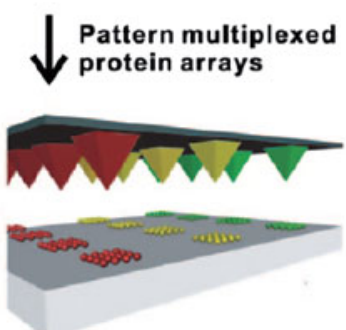

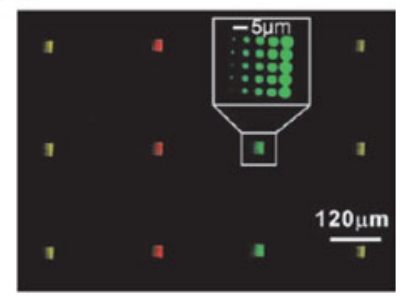

FIGURE 6. Scheme of the main steps for fabrication of multiplexed protein arrays. (a) Overview of PPL patterning process for fabrication of multiplexed protein arrays; (b) Si mold of three dye-conjugated proteins printed using inkjet printing; (c) Polymer pen array patterned onto Si mold using PPL; (d) Final product of the multiplexed protein arrays made by PPL with polymer pen array in (c). Figure reproduced with permission from Zheng et al. ${ }^{100}$ Copyright Wiley-VCH Verlag GmbH \& Co. KGaA.

were laterally printed utilizing SP1 and SP2 syringe pumps by Kloehn LTD. By modifying one pump for the lateral positioning of the syringe needle, which was placed only a few millimeters above the nitrocellulose paper, and another pump for the pressure driven flow, anti-streptavidin antibody were printed onto nitrocellulose paper and used to capture streptavidin-gold nano-particle conjugated antibody. In addition, it was shown that by controlling the flowrate of the antibody and the translational speed, it is possible to adjust the linewidth of the printed antibody; with the narrowest linewidth, $440 \mu \mathrm{m}$, achievable at a flow rate of $1.6 \mu \mathrm{L} \mathrm{min}{ }^{-1}$ and a translational speed of $3 \mathrm{~mm} \mathrm{~s}^{-1}$. Thus, by utilizing the common syringe pump, it was shown that lateral strip assays can be accomplished negating the use of expensive deposition equipments.

Inkjet printing has also emerged as one of the popular, rapid technologies used for delivery of small, controlled volumes of biomolecules onto substrates. ${ }^{2}$ Arrabito and Pignataro ${ }^{5}$ proposed the idea of using inkjet printing for dispensing molecular substances in a

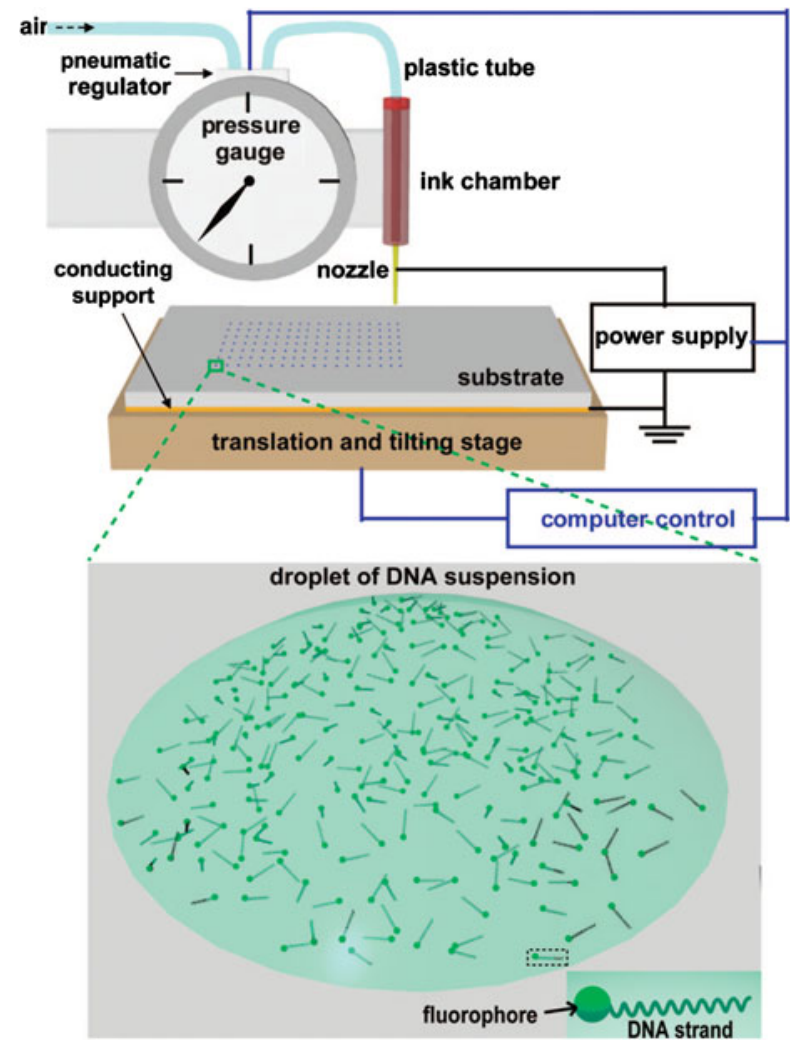

FIGURE 7. Schematic of the electrohydrodynamic jet (e-jet) printer. The e-jet printer can print single strand (ss) and double strand (ds) oligonucleotides onto substrates. Figure reprinted with permission from Park et al. ${ }^{74}$

microarray format. Here, an inkjet printer was used to deposit picoliters of D-glucose or D-glucose coupled with its inhibitor D-glucal onto glucose oxidase covalently linked to a functionalized silicon oxide support. Following this, horseradish peroxidase was added which when reacted with the glucose oxidase in the presence of oxygen formed a red color. Stewart et al. utilized a piezoelectric inkjet printer to deposit a pattern of antibodies onto a nylon membrane for fabrication of an immunoassay. ${ }^{82}$ Abe et al. ${ }^{1}$ used inkjet printing to fabricate lateral flow immunochromatographic devices. In their method, filter paper was soaked for a short period of time in solution of polystyrene in toluene. After this, an inkjet printer was used to deposit toluene droplets which etched patterns on the filter paper. ${ }^{1}$ Examples of the lateral flow immunochromatographic devices for detecting immunoglobin, IgG, were successfully demonstrated using a sandwich immunoassay. The limit of detection with the device was $10 \mu \mathrm{g} / \mathrm{L}$ and was able to detect within $20 \mathrm{~min}$.

For even higher resolution in the nanoscale range, Park et ll. $^{74}$ used an electrohydrodynamic jet (e-jet) printer to deposit DNA for applications in DNA microarray and biosensors. By utilizing electric fields, the e-jet printer was able to use micro/nanocapillary 
TABLE 3. Micro and nanofabricated patterning techniques.

\begin{tabular}{|c|c|c|c|}
\hline Description & Advantages & Required instrumentation/consumables & Applications \\
\hline $\mathrm{PPL}^{45}$ & $\begin{array}{l}\text { Multiplexed protein arrays, } \\
\text { nano-macroscopic resolution }\end{array}$ & $\begin{array}{l}\text { Si, inkjet printer, glycerol, oxygen } \\
\text { plasma, NSCRIPTOR }\end{array}$ & $\begin{array}{l}\text { Multiplexed patterning } \\
\text { of protein }\end{array}$ \\
\hline $\begin{array}{l}\text { Nanoarray from DPN } \\
\text { and } \text { TS }^{8}\end{array}$ & Label-free & $\begin{array}{l}\text { AFM tip, E-beam evaporation, } \\
\text { Au-Ag microgrid, NSCRIPTOR, } \\
\text { silicon wafer, mica }\end{array}$ & DNA sensor \\
\hline Lateral flow strips ${ }^{69}$ & $\begin{array}{l}\text { Minimal volume required for } \\
\text { deposition, ability to tune } \\
\text { line widths }\end{array}$ & $\begin{array}{l}\text { Syringe pump, nitrocellulose } \\
\text { membrane, gold nanoparticle, } \\
\text { sodium citrate }\end{array}$ & Immunoassays \\
\hline Inkjet printer for glucose ${ }^{5}$ & $\begin{array}{l}\text { Minimal volume required for } \\
\text { deposition }\end{array}$ & $\begin{array}{l}\text { Inkjet printer, nylon membrane, } \\
\text { D-glucose, silicon oxide } \\
\text { substrates }\end{array}$ & Glucose sensor \\
\hline Inkjet printer for antibodies ${ }^{1}$ & $\begin{array}{l}\text { LOD: } 10 \mu \mathrm{g} / \mathrm{L} \text {, silicon oxide } \\
\text { support }\end{array}$ & $\begin{array}{l}\text { Filter paper, polystyrene, } \\
\text { toluene, fatbrown RR dye }\end{array}$ & Immunoassay \\
\hline E-jet printer ${ }^{74}$ & High resolution (100 nm) & $\begin{array}{l}\text { E-jet printer, glycerin, } \\
\text { Si wafers, E-beam evaporation }\end{array}$ & DNA microarray/biosensors \\
\hline
\end{tabular}

glass nozzles to generate dots on the order of microsubmicrometer size (Fig. 7). Their limit of resolution using the e-jet printer was $100 \mathrm{~nm}$. In order to provide electrical contact with the ink, a thin layer of metal was deposited on the outer surface and inner surface closest to the tip. A pre-synthesized oligonucleotide fluorescently labeled with Alexa 546 was delivered via a metal coated nozzle in various patterns. More recently, Barton et al. $^{7}$ presented the idea of using a desktop system for e-jet printing. A summary of the patterning techniques described in this section is presented in Table 3.

\section{CONCLUSION}

We predict that the integration of novel materials with these low-cost fabrication technologies will provide some of the most promising developments in POC diagnostics in the coming decade. Specifically, through the application of these novel micro- and nano-fabrication techniques which enable 3D architectures, high resolution patterning, extremely low-cost substrate materials, enhanced sensitivity and signal to noise ratios, we can improve traditional approaches. Because many of these approaches are 'direct write' as opposed to 'top down' fabrication approaches, they offer unprecedented resolution as well as flexibility and easy extensibility on demand. For academic prototyping, these are attractive qualities in a technology platform. Finally, because of their low costs and relatively small tooling requirements, these technologies are readily adoptable by most academic laboratories. This will only serve to increase the rate of progress that we make on improving these promising young technologies. With these novels, rapid, and powerful techniques for fabrication of diagnostic chips combined with patterning of biomolecules onto substrates, the potential to use these technologies to help developing countries is very promising.

\section{OPEN ACCESS}

This article is distributed under the terms of the Creative Commons Attribution Noncommercial License which permits any noncommercial use, distribution, and reproduction in any medium, provided the original author(s) and source are credited.

\section{REFERENCES}

${ }^{1}$ Abe, K., K. Kotera, K. Suzuki, and D. Citterio. Inkjetprinted paperfluidic immuno-chemical sensing device. Anal. Bioanal. Chem. 398(2):885-893, 2010.

${ }^{2}$ Abe, K., K. Suzuki, and D. Citterio. Inkjet-printed microfluidic multianalyte chemical sensing paper. Anal. Chem. 80(18):6928-6934, 2008.

${ }^{3}$ Aroonrerk, N., A. Suksamrarn, and K. Kirtikara. A sensitive direct ELISA for detection of prostaglandin E2. J. Immunoassay Immunochem. 28(4):319-330, 2007.

${ }^{4}$ Arora, A., G. Simone, G. B. Salieb-Beugelaar, J. T. Kim, and A. Manz. Latest developments in micro total analysis systems. Anal. Chem. 82(12):4830-4847, 2010.

${ }^{5}$ Arrabito, G., and B. Pignataro. Inkjet printing methodologies for drug screening. Anal. Chem. 82(8):3104-3107, 2010.

${ }^{6}$ Barbulovic-Nad, I., M. Lucente, Y. Sun, M. Zhang, A. R. Wheeler, and M. Bussmann. Bio-microarray fabrication techniques-a review. Crit. Rev. Biotechnol. 26(4): 237-259, 2006.

${ }^{7}$ Barton, K., S. Mishra, K. A. Shorter, A. Alleyne, P. Ferreira, and J. Rogers. A desktop electrohydrodynamic jet printing system. Mechatronics 20(5):611-616, 2010.

${ }^{8}$ Baserga, A., M. ViganoÌ, C. S. Casari, S. Turri, A. Li Bassi, M. Levi, and C. E. Bottani. Au-Ag template stripped pattern for scanning probe investigations of 
DNA arrays produced by dip pen nanolithography. Langmuir 24(22):13212-13217, 2008.

${ }^{9}$ Betancourt, T., and L. Brannon-Peppas. Micro- and nanofabrication methods in nanotechnological medical and pharmaceutical devices. Int. J. Nanomedicine 1(4): 483-495, 2006.

${ }^{10}$ Bhattacharyya, A., and C. Klapperich. Design and testing of a disposable microfluidic chemiluminescent immunoassay for disease biomarkers in human serum samples. Biomed. Microdevices 9(2):245-251, 2007.

${ }^{11}$ Bhushan, B., and S. Matsui. Three-Dimensional Nanostructure Fabrication by Focused Ion Beam Chemical Vapor Deposition. Springer Handbook of Nanotechnology. Berlin, Heidelberg: Springer, pp. 211-229, 2010.

${ }^{12}$ Borenstein, J. T., E. J. Weinberg, B. K. Orrick, C. Sundback, M. R. Kaazempur-Mofrad, and J. P. Vacanti. Microfabrication of three-dimensional engineered scaffolds. Tissue Eng. 13(8):1837-1844, 2007.

${ }^{13}$ Bruzewicz, D. A., M. Reches, and G. M. Whitesides. Low-cost printing of poly(dimethylsiloxane) barriers to define microchannels in paper. Anal. Chem. 80(9):33873392, 2008

${ }^{14}$ Carlo, D. D., L. Y. Wu, and L. P. Lee. Dynamic single cell culture array. Lab Chip 6(11):1445-1449, 2006.

${ }^{15}$ Carrilho, E., A. W. Martinez, and G. M. Whitesides. Understanding wax printing: a simple micropatterning process for paper-based microfluidics. Anal. Chem. 81(16):7091-7095, 2009.

${ }^{16}$ Chen, C. S., D. N. Breslauer, J. I. Luna, A. Grimes, W. C. Chin, L. P. Lee, and M. Khine. Shrinky-Dink microfluidics: 3D polystyrene chips. Lab Chip 8(4):622624, 2008.

${ }^{17}$ Chen, X., D. C. Chang, and C. Liu. On-line cell lysis and DNA extraction on a microfluidic biochip fabricated by microelectromechanical system technology. Electrophoresis 29:1844-1851, 2008.

${ }^{18}$ Cheng, C. M., A. W. Martinez, J. Gong, C. R. Mace, S. T. Phillips, E. Carrilho, K. A. Mirica, and G. M. Whitesides. Paper-based ELISA. Angew. Chem. Int. Ed. Engl. 49(28):4771-4774, 2010.

${ }^{19}$ Cheung, Y. K., B. M. Gillette, M. Zhong, S. Ramcharan, and S. K. Sia. Direct patterning of composite biocompatible microstructures using microfluidics. Lab Chip 7(5):574-579, 2007.

${ }^{20}$ Chin, C. D. Biotechnology for global health: solutions for the developing world. Consilience - J. Sustain. Develop. (1): 1-12, 2008.

${ }^{21}$ Chin, C. D., V. Linder, and S. K. Sia. Lab-on-a-chip devices for global health: past studies and future opportunities. Lab Chip 7(1):41-57, 2007.

${ }^{22}$ Choi, J. H., R. Ganesan, D. K. Kim, C. H. Jung, I. T. Hwang, Y. C. Nho, J. M. Yun, and J. B. Kim. Patterned immobilization of biomolecules by using ion irradiation-induced graft polymerization. J. Polym. Sci. A: Polym. Chem. 47(22):6124-6134, 2009.

${ }^{23}$ Chován, T., and A. Guttman. Microfabricated devices in biotechnology and biochemical processing. Trends Biotechnol. 20(3):116-122, 2002.

${ }^{24}$ Coltro, W. K. T., D. P. de Jesus, J. A. F. da Silva, C. L. do Lago, and E. Carrilho. Toner and paper-based fabrication techniques for microfluidic applications. Electrophoresis 31(15):2487-2498, 2010.

${ }^{25}$ de Boer, M. J., R. W. Tjerkstra, J. W. Berenschot, H. V. Jansen, G. J. Burger, J. G. E. Gardeniers, M. Elwenspoek, and A. van den Berg. Micromachining of buried micro channels in silicon. J. Microelectromech. Syst. 9(1):94-103, 2000.

${ }^{26}$ Dittrich, P. S., and A. Manz. Lab-on-a-chip: microfluidics in drug discovery. Nat. Rev. Drug. Discov. 5(3):210-218, 2006.

${ }^{27}$ Engvall, E., and P. Perlmann. Enzyme-linked immunosorbent assay (ELISA) quantitative assay of immunoglobulin G. Immunochemistry 8(9):871-874, 1971.

${ }^{28}$ Erickson, K. A., and P. Wilding. Evaluation of a novel point-of-care system, the i-STAT portable clinical analyzer. Clin. Chem. 39:283-287, 1993

${ }^{29}$ Focke, M., D. Kosse, C. Muller, H. Reinecke, R. Zengerle, and F. von Stetten. Lab-on-a-foil: microfluidics on thin and flexible films. Lab Chip 10(11):1365-1386, 2010.

${ }^{30} \mathrm{Fu}$, C. C., A. Grimes, M. Long, C. G. L. Ferri, B. D. Rich, S. Ghosh, L. P. Lee, A. Gopinathan, and M. Khine. Tunable nanowrinkles on shape memory polymer sheets. Adv. Mater. 21(44):4472-4476, 2009.

${ }^{31} \mathrm{Fu}, \mathrm{C} . \mathrm{C}$., G. Ossato, M. Long, M. Digman, L. P. Lee, E. Gratton, and M. Khine. Bimetallic nanopetals for thousand-fold fluorescence enhancements. Appl. Phys. Lett. 97(20):203101-1-203101-3, 2010.

${ }^{32}$ Gao, H. W., J. C. Yang, J. Y. Lin, A. D. Stuparu, M. H. Lee, M. Mrksich, and T. W. Odom. Using the angle-dependent resonances of molded plasmonic crystals to improve the sensitivities of biosensors. Nano Lett. 10(7): 2549-2554, 2010

${ }^{33}$ Geissler, M., E. Roy, G. A. Diaz-Quijada, J.-C. Galas, and T. Veres. Microfluidic patterning of miniaturized DNA arrays on plastic substrates. ACS Appl. Mater. Interfaces 1(7):1387-1395, 2009.

${ }^{34}$ Geissler, M., and Y. Xia. Patterning: principles and some new developments. Adv. Mater. 16(15):1249-1269, 2004.

${ }^{35}$ Gratton, S. E. A., S. S. Williams, M. E. Napier, P. D. Pohlhaus, Z. Zhou, K. B. Wiles, B. W. Maynor, C. Shen, T. Olafsen, E. T. Samulski, et al. The pursuit of a scalable nanofabrication platform for use in material and life science applications. Acc. Chem. Res. 41(12):1685-1695, 2008.

${ }^{36}$ Grimes, A., D. N. Breslauer, M. Long, J. Pegan, L. P. Lee, and M. Khine. Shrinky-Dink microfluidics: rapid generation of deep and rounded patterns. Lab Chip 8(1):170-172, 2008.

${ }^{37}$ Handali, S., M. Klarman, A. N. Gaspard, X. F. Dong, R. LaBorde, J. Noh, Y.-M. Lee, S. Rodriguez, A. E. Gonzalez, H. H. Garcia, et al. Development and evaluation of a magnetic immunochromatographic test to detect Taenia solium, which causes taeniasis and neurocysticercosis in humans. Clin. Vaccine Immunol. 17(4):631-637, 2010.

${ }^{38}$ Hansen, C. L., S. Classen, J. M. Berger, and S. R. Quake. A microfluidic device for kinetic optimization of protein crystallization and in situ structure determination. J. Am. Chem. Soc. 128(10):3142-3143, 2006.

${ }^{39}$ Heckele, M., W. Bacher, and K. D. Müller. Hot embossing - The molding technique for plastic microstructures. Microsyst. Technol. 4(3):122-124, 1998.

${ }^{40}$ Heckele, M., and W. K. Schomburg. Review on micro molding of thermoplastic polymers. J. Micromech. Microeng. 14(3):R1-R14, 2004.

${ }^{41}$ Hegner, M., P. Wagner, and G. Semenza. Ultralarge atomically flat template-stripped Au surfaces for scanning probe microscopy. Surf. Sci. 291(1-2):39-46, 1993.

${ }^{42}$ Henzie, J., M. H. Lee, and T. W. Odom. Multiscale patterning of plasmonic metamaterials. Nat. Nano. 2(9):549$554,2007$. 
${ }^{43}$ Hidber, P. C., P. F. Nealey, W. Helbig, and G. M. Whitesides. New strategy for controlling the size and shape of metallic features formed by electroless deposition of copper: microcontact printing of catalysts on oriented polymers, followed by thermal shrinkage. Langmuir 12(21):5209-5215, 1996.

${ }^{44}$ Hierlemann, A., O. Brand, C. Hagleitner, and H. Baltes. Microfabrication techniques for chemical/biosensors. Proc. IEEE 91(6):839-863, 2003.

${ }^{45}$ Huo, F., Z. Zheng, G. Zheng, L. R. Giam, H. Zhang, and C. A. Mirkin. Polymer pen lithography. Science 321(5896):1658-1660, 2008.

${ }^{46}$ Khademhosseini, A., R. Langer, J. Borenstein, and J. P. Vacanti. Microscale technologies for tissue engineering and biology. Proc. Natl. Acad. Sci. USA 103(8): 2480-2487, 2006.

${ }^{47}$ Khine, M., A. Lau, C. Ionescu-Zanetti, J. Seo, and L. P. Lee. A single cell electroporation chip. Lab Chip 5(1):38-43, 2005.

${ }^{48}$ Koerner, T., L. Brown, R. Xie, and R. D. Oleschuk. Epoxy resins as stamps for hot embossing of microstructures and microfluidic channels. Sens. Actuators B: Chemical 107(2):632-639, 2005.

${ }^{49}$ Landry, M. L. Developments in immunologic assays for respiratory viruses. Clin. Lab. Med. 29(4):635-647, 2009.

${ }^{50}$ Lee, D.-S., S. H. Park, H. Yang, K.-H. Chung, T. H. Yoon, S.-J. Kim, K. Kim, and Y. T. Kim. Bulkmicromachined submicroliter-volume PCR chip with very rapid thermal response and low power consumption. $L a b$ Chip 4(4):401-407, 2004.

${ }^{51}$ Lee, D.-S., M.-H. Wu, U. Ramesh, C.-W. Lin, T.-M. Lee, and P.-H. Chen. A novel real-time PCR machine with a miniature spectrometer for fluorescence sensing in a micro liter volume glass capillary. Sens. Actuators B: Chemical 100(3):401-410, 2004.

${ }^{52}$ Lee, M. H., M. D. Huntington, W. Zhou, J.-C. Yang, and T. W. Odom. Programmable soft lithography: solventassisted nanoscale embossing. Nano Lett., 2010.

${ }^{53}$ Lee, S., S.-S. Bae, G. Medeiros-Ribeiro, J. J. Blackstock, S. Kim, D. R. Stewart, and R. Ragan. Scanning tunneling microscopy of template-stripped $\mathrm{Au}$ surfaces and highly ordered self-assembled monolayers. Langmuir 24(12): 5984-5987, 2008.

${ }^{54} \mathrm{Li}$, X., J. Tian, and W. Shen. Thread as a versatile material for low-cost microfluidic diagnostics. ACS Appl. Mater. Interfaces 2(1):1-6, 2010.

${ }^{55}$ Liu, G. L., Y. Yin, S. Kunchakarra, B. Mukherjee, D. Gerion, S. D. Jett, D. G. Bear, J. W. Gray, A. P. Alivisatos, L. P. Lee, L. P., et al. A nanoplasmonic molecular ruler for measuring nuclease activity and DNA footprinting. Nat. Nano 1(1):47-52, 2006.

${ }^{56}$ Liu, Y., D. Yang, T. Yu, and X. Jiang. Incorporation of electrospun nanofibrous PVDF membranes into a microfluidic chip assembled by PDMS and scotch tape for immunoassays. Electrophoresis 30(18):3269-3275, 2009.

${ }^{57}$ Lu, H., M. A. Schmidt, and K. F. Jensen. A microfluidic electroporation device for cell lysis. Lab Chip 5(1):23-29, 2005.

${ }^{58}$ Lu, Y., W. Shi, L. Jiang, J. Qin, and B. Lin. Rapid prototyping of paper-based microfluidics with wax for low-cost, portable bioassay. Electrophoresis 30(9):1497-1500, 2009.

${ }^{59}$ Lu, Y., W. Shi, J. Qin, and B. Lin. Fabrication and characterization of paper-based microfluidics prepared in nitrocellulose membrane by wax printing. Anal. Chem. 82(1):329-335, 2009.
${ }^{60}$ Luo, Y., F. Yu, and R. N. Zare. Microfluidic device for immunoassays based on surface plasmon resonance imaging. Lab Chip 8(5):694-700, 2008.

${ }^{61}$ Lyashchenko, K. P., M. Singh, R. Colangeli, and M. L. Gennaro. A multi-antigen print immunoassay for the development of serological diagnosis of infectious diseases. J. Immunol. Methods 242(1-2):91-100, 2000.

${ }^{62}$ Martinez, A. W., S. T. Phillips, M. J. Butte, and G. M. Whitesides. Patterned paper as a platform for inexpensive, low-volume, portable bioassays. Angew. Chem. Int. Ed. Engl. 46(8):1318-1320, 2007.

${ }^{63}$ Martinez, A. W., S. T. Phillips, Z. Nie, C.-M. Cheng, E. Carrilho, B. J. Wiley, and G. M. Whitesides. Programmable diagnostic devices made from paper and tape. Lab Chip 10(19):2499-2504, 2010.

${ }^{64}$ Martinez, A. W., S. T. Phillips, and G. M. Whitesides. Three-dimensional microfluidic devices fabricated in layered paper and tape. Proc. Natl. Acad. Sci. USA 105(50):19606-19611, 2008.

${ }^{65}$ McDonald, J. C., D. C. Duffy, J. R. Anderson, D. T. Chiu, H. Wu, O. J. Schueller, and G. M. Whitesides. Fabrication of microfluidic systems in poly(dimethylsiloxane). Electrophoresis 21(1):27-40, 2000.

${ }^{66}$ McKenzie, K. G., L. K. Lafleur, B. R. Lutz, and P. Yager. Rapid protein depletion from complex samples using a bead-based microfluidic device for the point of care. Lab Chip 9(24):3543-3548, 2009.

${ }^{67}$ Monaghan, P. B., K. M. McCarney, A. Ricketts, R. E. Littleford, F. Docherty, W. E. Smith, D. Graham, and J. M. Cooper. Bead-based DNA diagnostic assay for chlamydia using nanoparticle-mediated surface-enhanced resonance Raman scattering detection within a lab-on-achip format. Anal. Chem. 79(7):2844-2849, 2007.

${ }^{68}$ Mukhopadhyay, R. When PDMS isn't the best. Anal. Chem. 79(9):3248-3253, 2007.

${ }^{69}$ Nash, M. A., J. M. Hoffman, D. Y. Stevens, A. S. Hoffman, P. S. Stayton, and P. Yager. Laboratory-scale protein striping system for patterning biomolecules onto paper-based immunochromatographic test strips. Lab Chip 10(17):2279-2282, 2010.

${ }^{70}$ Nath, P., D. Fung, Y. A. Kunde, A. Zeytun, B. Branch, and G. Goddard. Rapid prototyping of robust and versatile microfluidic components using adhesive transfer tapes. Lab Chip 10(17):2286-2291, 2010.

${ }^{71}$ Nguyen, D., D. Taylor, K. Qian, N. Norouzi, J. Rasmussen, S. Botzet, M. Lehmann, K. Halverson, and M. Khine. Better shrinkage than Shrinky-Dinks. Lab Chip 10(12):1623-1626, 2010.

${ }^{72} \mathrm{Nie}, \mathrm{Z}$., and E. Kumacheva. Patterning surfaces with functional polymers. Nat. Mater. 7(4):277-290, 2008.

${ }^{73}$ Novak, L., P. Neuzil, J. Pipper, Y. Zhang, and S. Lee. An integrated fluorescence detection system for lab-on-a-chip applications. Lab Chip 7(1):27-29, 2007.

${ }^{74}$ Park, J. U., J. H. Lee, U. Paik, Y. Lu, and J. A. Rogers. Nanoscale patterns of oligonucleotides formed by electrohydrodynamic jet printing with applications in biosensing and nanomaterials assembly. Nano Lett. 8(12): 4210-4216, 2008.

${ }^{75}$ Pham, D. T., and R. S. Gault. A comparison of rapid prototyping technologies. Int. J. Mach. Tools Manuf. 38(10-11):1257-1287, 1998

${ }^{76}$ Piner, R. D., J. Zhu, F. Xu, S. Hong, and C. A. Mirkin. "Dip-Pen" nanolithography. Science 283(5402):661-663, 1999. 
${ }^{77}$ Reches, M., K. A. Mirica, R. Dasgupta, M. D. Dickey, M. J. Butte, and G. M. Whitesides. Thread as a matrix for biomedical assays. ACS Appl. Mater. Interfaces 2(6): 1722-1728, 2010.

${ }^{78}$ Rötting, O., W. Röpke, H. Becker, and C. Gärtner. Polymer microfabrication technologies. Microsyst. Technol. 8(1):32-36, 2002

${ }^{79}$ Sia, S. K., V. Linder, B. A. Parviz, A. Siegel, and G. M. Whitesides. An integrated approach to a portable and low-cost immunoassay for resource-poor settings. Angew. Chem. Int. Ed. 43(4):498-502, 2004.

${ }^{80}$ Sollier, K., C. A. Mandon, K. A. Heyries, L. J. Blum, and C. A. Marquette. "Print-n-Shrink" technology for the rapid production of microfluidic chips and protein microarrays. Lab Chip 9(24):3489-3494, 2009.

${ }^{81}$ Stewart, M. E., N. H. Mack, V. Malyarchuk, J. A. N. T. Soares, T.-W. Lee, S. K. Gray, R. G. Nuzzo, and J. A. Rogers. Quantitative multispectral biosensing and 1D imaging using quasi-3D plasmonic crystals. Proc. Natl. Acad. Sci. 103(46):17143-17148, 2006.

${ }^{82}$ Stewart, T. N., B. E. Pierson, R. Aggarwal, and R. J. Narayan. Piezoelectric inkjet printing of a cross-hatch immunoassay on a disposable nylon membrane. Biotechnol. J. 4(2):206-209, 2009.

${ }^{83}$ Sudarsan, A. P., J. Wang, and V. M. Ugaz. Thermoplastic elastomer gels: an advanced substrate for microfluidic chemical analysis systems. Anal. Chem. 77(16):5167-5173, 2005.

${ }^{84}$ Taylor, D., D. Dyer, V. Lew, and M. Khine. Shrink film patterning by craft cutter: complete plastic chips with high resolution/high-aspect ratio channel. Lab Chip 10(18): 2472-2475, 2010.

${ }^{85}$ Voldman, J., M. L. Gray, and M. A. Schmidt. Microfabrication in biology and medicine. Ann. Rev. Biomed. Eng. 1(1):401-425, 1999.

${ }^{86}$ Wang, W., S. Zhao, and T. Pan. Lab-on-a-print: from a single polymer film to three-dimensional integrated microfluidics. Lab Chip 9:1133-1137, 2009.

${ }^{87}$ Wiley, B. J., D. Qin, and Y. Xia. Nanofabrication at high throughput and low cost. ACS Nano 4(7):3554-3559, 2010.

${ }^{88}$ Wood, D. K., D. M. Weingeist, S. N. Bhatia, and B. P. Engelward. Single cell trapping and DNA damage analysis using microwell arrays. Proc. Natl. Acad. Sci. 107(22):10008-10013, 2010.

${ }^{89}$ Worgull, M., and M. Heckele. New aspects of simulation in hot embossing. Microsyst. Technol. 10(5):432-437, 2004.

${ }^{90}$ Wu, S., S. R. Park, and X. S. Ling. Lithography-free formation of nanopores in plastic membranes using laser heating. Nano Lett. 6(11):2571-2576, 2006.

${ }^{91}$ Xiang, Q., B. Xu, and D. Li. Miniature real time PCR on chip with multi-channel fiber optical fluorescence detection module. Biomed. Microdevices 9(4):443-449, 2007.

${ }^{92}$ Yager, P., G. J. Domingo, and J. Gerdes. Point-of-care diagnostics for global health. Ann. Rev. Biomed. Eng. 10:107-144, 2008.

${ }^{93}$ Yager, P., T. Edwards, E. Fu, K. Helton, K. Nelson, M. R. Tam, and B. H. Weigl. Microfluidic diagnostic technologies for global public health. Nature 442(7101): 412-418, 2006

${ }^{94}$ Yang, J. C., H. W. Gao, J. Y. Suh, W. Zhou, M. H. Lee, and T. W. Odom. Enhanced optical transmission mediated by localized plasmons in anisotropic, threedimensional nanohole arrays. Nano Lett. 10(8):3173-3178, 2010.

${ }^{95}$ Yao, J., A. P. Le, S. K. Gray, J. S. Moore, J. A. Rogers, and R. G. Nuzzo. Functional nanostructured plasmonic materials. Adv. Mater. 22(10):1102-1110, 2010.

${ }^{96}$ Yuen, P. K., and V. N. Goral. Low-cost rapid prototyping of flexible microfluidic devices using a desktop digital craft cutter. Lab Chip 10(3):384-387, 2010.

${ }^{97}$ Zare, R. N., and S. Kim. Microfluidic platforms for single-cell analysis. Ann. Rev. Biomed. Eng. 12:187-201, 2010.

${ }^{98}$ Zhao, S., H. Cong, and T. Pan. Direct projection on dryfilm photoresist (DP2): do-it-yourself three-dimensional polymer microfluidics. Lab Chip 9(8):1128-1132, 2009.

${ }^{99}$ Zhao, X. M., Y. Xia, D. Qin, and G. M. Whitesides. Fabrication of polymeric microstructures with high aspect ratios using shrinkable polystyrene films. Adv. Mater. 9(3):251-254, 1997.

${ }^{100}$ Zheng, Z., W. L. Daniel, L. R. Giam, F. Huo, A. J. Senesi, G. Zheng, and C. A. Mirkin. Multiplexed protein arrays enabled by polymer pen lithography: addressing the inking challenge. Angew. Chem. Int. Ed. Engl. 48(41):7626-7629, 2009. 